\title{
Enhancing visible-light-enhanced photoelectrochemical activity of BiOl microspheres for 4-chlorophenol detection by promoting with Bi surface plasmon resonance (SPR) and multi-walled carbon nanotubes
}

\author{
Liqin Yang ${ }^{1} \cdot$ Zhiju Zhao $^{1}$ (D) Zhenyu Cai $^{2}$
}

Received: 27 March 2020 / Accepted: 9 June 2020 / Published online: 15 June 2020

c) Springer Nature Switzerland AG 2020

\begin{abstract}
This work interprets Bi surface plasmon resonance (SPR) and Multi-walled carbon nanotubes, which promote BiOI microspheres (MWCNT/Bi-BiOl) and put into synthesis in virtue of one-stage solvothermal method. The measurement of the combination structure with morphology is achieved in virtue of a range of physical characteristics. Moreover, as the photoelectrochemical (PEC) tests indicate, MWCNT/Bi-BiOl features higher PEC properties compared to those of pure $\mathrm{BiOl}$ for the reason of the active SPR influence of Bi nanoparticles as well as the excellent electrical conductivity of MWCNT. Besides, MWCNT/Bi-BiOI is characterized by superior absorption of visible light together with a lower-electron recombination ratio. Thereby, MWCNT/Bi-BiOI-based PEC sensors have been made up to detect 4-chlorophenol (4-CP). They display wide linear within the range of $1-12,800 \mathrm{ng} \mathrm{mL}^{-1}$, based on the high sensing 4-CP stability. As a result, the PEC sensor harbors an excellent potential application in the detection of 4-CP.
\end{abstract}

Keywords Surface plasmon resonance - Multi-walled carbon nanotubes · Photoelectrochemical · Detection . 4-Chlorophenol

\section{Introduction}

4-Chlorophenol (4-CP) is an organochlorine widely used in chemical and pharmaceutical, coking, steelmaking and construction [1-3]. However, 4-CP is highly toxic and can harm human health and life [3]. Accordingly, an urgent demand is existing for the monitoring the water condition of $4-\mathrm{CP}$ concentrations.

Therefore, developing rapid and sensitive detection methods for 4-CP can promote the ability to launch preventive for this environmental issue in a timely manner. There are several methods to detect $4-\mathrm{CP}$, such as gas chromatography [4], high-performance liquid chromatography [5], electrochemical detection [6]. Every strategy has distinct advantages for detection of 4-CP, yet suffers significantly from time-consuming preparation, toxic solvents, and high equipment cost. Therefore, accurate, ultrasensitive, easy to use, and low-cost detection methods still of critical urgency for tracking 4-CP pollution.

At present, Photoelectrochemical (PEC) detection has been successfully used to detect multiple drugs as a reliable and rapidly evolving technology [7] since the excitation source and the electrochemical detection signal are completely separated, background noise can be reduced and

Electronic supplementary material The online version of this article (https://doi.org/10.1007/s42452-020-3027-2) contains supplementary material, which is available to authorized users.

Zhiju Zhao, zhaozhiju@hotmail.com; $\triangle$ Zhenyu Cai, caizy1963@163.com|'College of Chemistry and Chemical Engineering, Xingtai University, Xingtai 054001, Hebei, China. ${ }^{2}$ Xingtai University, Xingtai 054001, Hebei, China. 
sensitivity can be improved $[8,9]$. The measurement of the PEC method is based on the optical and electrical properties of semiconductor materials $[10,11]$. Therefore, the development of semiconductor materials that are easy to synthesize and have a broad optical response is essential for manufacturing ultrasensitive PEC methods.

Bismuth-system oxides, which is composed of $6 \mathrm{~s} \mathrm{Bi}$ orbital with $2 p$ oxygen orbital, displays strong optical activity and excellent electrical properties $[12,13]$. The reason accounting for why $\mathrm{BiOX}(\mathrm{X}=\mathrm{Cl}, \mathrm{I}, \mathrm{Br})$ has attracted widely attention in PEC field is because of its abundance, low toxicity and inexpensiveness [14-16]. Among them, BiOl have the narrowest band gap $(\sim 1.8 \mathrm{eV})$, and it is a semiconductor of $p$ type equipped with a large visible light-absorption capacity $[17,18]$. In addition, BiOI harbores has superior photocatalytic activity due to the unique layered structure [19]. However, BiOl belongs to the narrow band gap semiconductor, which results the sped-up recombination of photogenerated electrons as well as holes $[20,21]$. The combination of some else semiconductor-based materials and $\mathrm{BiOl}$ accelerates electron-hole pair division in virtue of improving electron transfer efficiency to increase energy conversion efficiency [22-25].

Metal bismuth (Bi), which is characterized by some lowexpense and broad-source advantages is a kind of semimetal, with narrow-band gap and small-carrier-effective mass, as well as high anisotropic Fermi surface with low carrier density [26, 27]. Moreover, being the precious metals, Bi takes on the same features of surface plasmon resonance (SPR), enhanced utilization of electron-hole pairs as it is often employed as the combined electron performer with conductor [28]. Multi-walled carbon nanotubes (MWCNTs) are attracting attention due to their special structure and unique electrical properties [29]. In addition, multi-walled carbon nanotubes have higher electron conductivity. It acts as an efficient electron acceptor to improve the photoinduced charge transfer that enhances the PEC performance [30]. Therefore, they have potential application prospects in the field of PEC.

In this manuscript, it reports the PEC sensor of the 4-CP based on MWCNT/Bi-BiOI. The PEC experiments show that 20-MWCNT/Bi-BiOI-0.5 harbors wonderful PEC performance, meanwhile exhibiting a broad linear range, low detection limit $\left(0.65 \mathrm{ng} \mathrm{mL}^{-1}, \mathrm{~S} / \mathrm{N}=3\right)$ as well as superior selectivity of 4-CP detection. According to the results, the sensor on the basis of 20-MWCNT/Bi-BiOI-0.5 holds superior application prospects within practically useful $4-\mathrm{CP}$ detection.

\section{Experiment section}

\subsection{Correspondence regent}

All the reagents were of analysis-based grade free of further purification. Sinopharm Chemical Reagent Co., Ltd (www.sinoreagent.com) was the supplier for Bismuth nitrate pentahydrate $\left(\mathrm{Bi}\left(\mathrm{NO}_{3}\right)_{3} \cdot 5 \mathrm{H}_{2} \mathrm{O}\right)$, potassiumiodide $(\mathrm{KI})$, MWCNTs (diameters $<8 \mathrm{~nm}$ and $0.5-2 \mu \mathrm{m}$ length), ethylene glycol (EG), glucose, acetone and ethanol.

\subsection{Prepared-item for the MWCNT/Bi-BiOI heterojunction}

The MWCNT/Bi-BiOl heterojunction was given the preparations from one-stage hydrothermal approach: proper $\mathrm{KI}$ and glucose used as individual addition into the solution of $40 \mathrm{ml} \mathrm{EG}$. Next, some proper $\mathrm{Bi}\left(\mathrm{NO}_{3}\right)_{3} .5 \mathrm{H}_{2} \mathrm{O}$, glucose and MWCNT got added to $\mathrm{KI}$ solution and continuous with 30 -min stirring. The mixed solution was put into $24 \mathrm{~h}$ heat at $160^{\circ} \mathrm{C}$ in Teflon-lined autoclave $(100 \mathrm{~mL})$. At last, after the hydrothermal process, product were collected, washed with deionized water and ethanol several times and dried at $60^{\circ} \mathrm{C}$, these ready-made materials conforming that the material amount will be on show in Table S1.

\subsection{Characterization}

X-ray diffraction (XRD) patterns were committed to a Bruker D8 Advance diffractometer based on Cu Ka radiation. X-ray photoelectron spectra were obtained by X-ray photoelectron spectroscope (XPS, ESCALAB 250Xi). The testament of diffuse reflection spectra (DRS) for materials was made by UV-Vis spectrophotomer by taking reference of $\mathrm{BaSO}_{4}$. The samples' morphology was featured with electron microscopy (SEM) and Transmission electron microscopy (TEM).

\subsection{Photoelectrochemical experiments}

Photocurrent tests were measured with the three-electrode system in an electrolytic cell (CHI 660E). Pt wire and SCE (saturated calomel electrode) acted as counter electrode and reference electrode, respectively. The working electrode referred to the indium tin oxide (ITO) glass. The light source referred to Xenon lamp (PLS-SXE $\left.300,100 \mathrm{~mW} \mathrm{~cm}{ }^{-2}, \lambda \geq 420 \mathrm{~nm}\right)$. From frequency $(\mathrm{Hz})$ $1-1,000,000$ in PBS $(0.1 \mathrm{M}, \mathrm{PH}=7)$, we carried out the electrochemical impedance spectroscopy (EIS). Using water, acetone and ethanol, the indium tin oxide (ITO) glass $(10 \times 15 \mathrm{~mm})$ were cleaned respectively for $5 \mathrm{~min}$. 
Subsequently, in mixed chitosan and ethanol liquid $(0.5 \mathrm{~mL}), 3 \mathrm{mg}$ catalyst powders were distributed for the formation of homogeneous suspension. Subsequently, ITO electrode $\left(0.5 \mathrm{~cm}^{2}\right)$ was coated with $20 \mu \mathrm{L}$ suspensions.

\section{Results and discussions}

\subsection{Materials choice}

The investigation on the phase of all the synthesized materials is made by XRD. Figure 1a reveals that as for all of the Bi-BiOl composites, all of diffraction peaks of BiOI (JCPDS 10-0445) are under clear observation. The three un-striking peaks respectively at around $27.16^{\circ}, 37.95^{\circ}$ and $39.62^{\circ}$ were corresponded to the (012), (104), (110) of Bi (JCPDS44-1246). In Fig. 1b, for MWCNT/Bi-BiOI, after adding MWCNT, the characteristic peaks of $\mathrm{BiPO}_{4}$ and $\mathrm{BiOI}$ did not change significantly. More than that, the featured peaks respectively at around $26.01^{\circ}$ are corresponded to the (002) of MWCNT. According to the results display, the synthesized materials can be classified as MWCNT/Bi-BiOI in a perfect way.

Further evaluate the PEC properties of all synthetic materials through photocurrent reaction experiments (Fig. 1c, d), which was redone 4 times at the frequency of the every regular $20 \mathrm{~s}$ in $0.1 \mathrm{M}$ PBS under the irradiation of visible light. All materials' photocurrents are enhanced with the irradiation of visible light. The photocurrent value of $\mathrm{Bi}-\mathrm{BiOI}-0.5$ is significantly higher compared to these of $\mathrm{BiOI}, \mathrm{Bi}-\mathrm{BiO}-0.2$ and Bi-BiOl-0.7 (Fig. 1C). Furthermore, 20-MWCNT/Bi-BiOI-0.5 has higher photocurrent than 10-MWCNT/Bi-BiOl-0.5 and 30-MWCNT/Bi-BiOl-0.5 in Fig. $1 \mathrm{~d}$. Consequently, compared to other materials, the photocurrent of 20-MWCNT/BPI-5\% is higher.

Electrochemical impedance spectra (EIS) were utilized for further evaluation on the electron transfer kinetics harbored by the whole composites (Figure S1A-B). The EIS is made up of a pressed semicircle part as well as a linear part. The electron transfer resistance (Rct) is
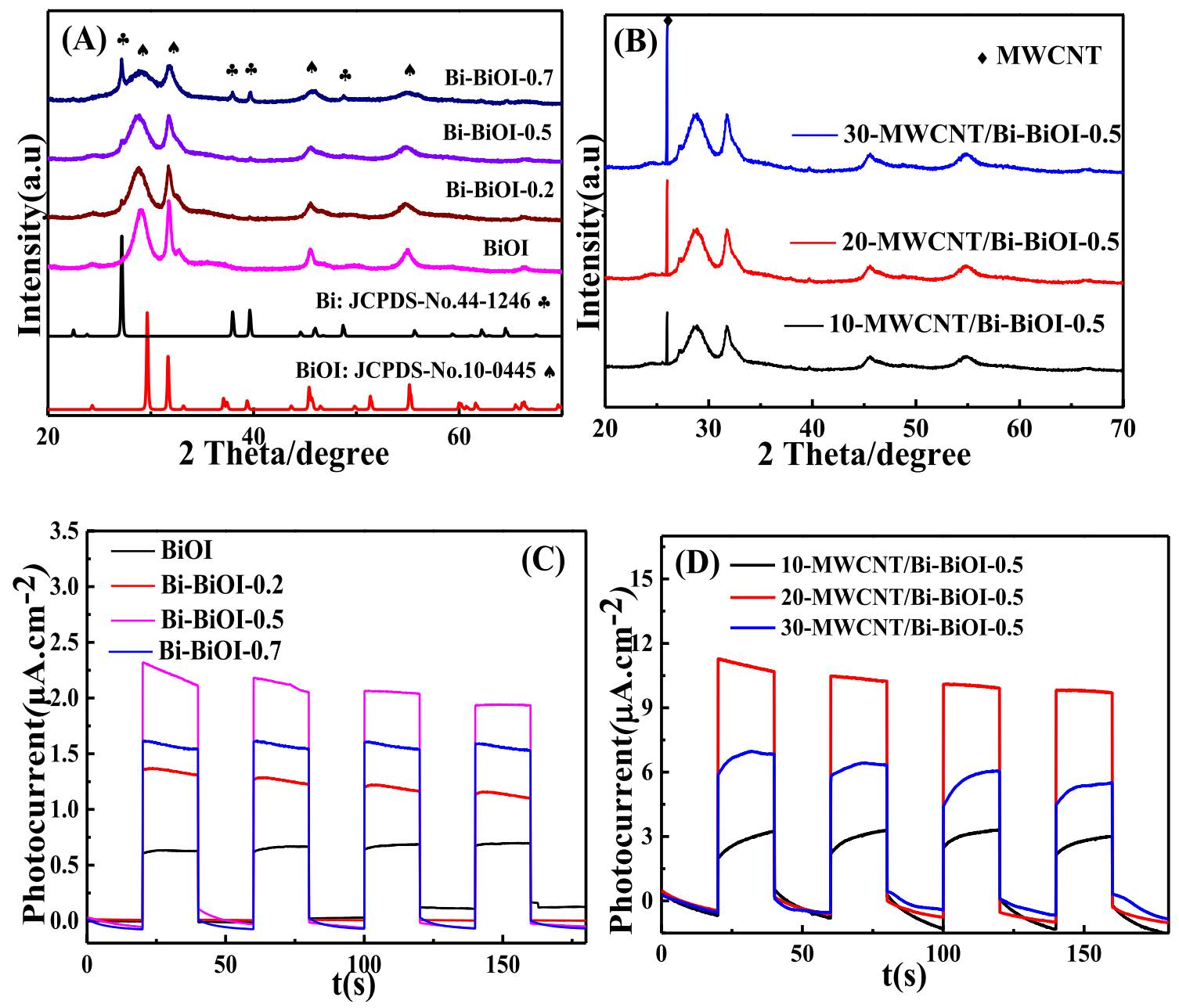

Fig. 1 XRD spectrum of the MWCNT/Bi-BiOI $(\mathbf{a}, \mathbf{b})$ composites, photocurrent responses $(\mathbf{c}$, $\mathbf{d})$ of the all materials in $0.1 \mathrm{M}$ PBS 
quantified with the help of the semicircle diameter [13]. And it can be ranked as: $\mathrm{BiOl}>\mathrm{Bi}-\mathrm{BiOl}-0.2>\mathrm{Bi}-\mathrm{BiOl}-0.7>\mathrm{Bi}-$ BiOl-0.5 > 10-MWCNT/Bi-BiOl-0.5 > 30-MWCNT/BiBiOl-0.5 > 20-MWCNT/Bi-BiOl-0.5. And 20-MWCNT/BiBiOl-0.5 shows the lowest impedance among composites. The result indicates that MWCNT and Bi provide rapid electron transfer capability and remarkable conductivity $[27,30]$. Drawing from the above results, 20-MWCNT/Bi$\mathrm{BiOI}-0.5$ was selected to be further investigated.

\subsection{The characterization of 20-MWCNT/Bi-BiOI-0.5}

The detailed chemical composition of 20-MWCNT/BiBiOl-0.5 was investigated by the XPS spectra in Fig. 2a-d. The C1s XPS is shown in Fig. 2b. The four peaks located at $284.12 \mathrm{eV}, 284.6 \mathrm{eV}, 285.69 \mathrm{eV}$ and $288.48 \mathrm{eV}$, which are ascribed to $\mathrm{C}-\mathrm{O}, \mathrm{C}-\mathrm{C}=\mathrm{C}, \mathrm{C}=\mathrm{O}$ and $\mathrm{O}-\mathrm{C}=\mathrm{O}$, respectively [30]. As Fig. 2c shows, the peaks harbored by $158.8 \mathrm{eV}$ and $164.2 \mathrm{eV}$ are distributed toward $\mathrm{Bi} 4 \mathrm{f}_{7 / 2}$ as well as $B i \mathrm{f}_{5 / 2}$ in the standard $\mathrm{Bi}^{3+}$ [31]. The peak of $156.3 \mathrm{eV}$ and $161.7 \mathrm{eV}$ result from zero valence state of metallic $\mathrm{Bi}$ [32]. Two peaks about $630.2 \mathrm{eV}$ and $618.5 \mathrm{eV}$ are ascribed to $3 \mathrm{~d}_{3 / 2}$ and $3 d_{5 / 2}$ of BiOl, which correspond to $\mathrm{I}^{-1}$ (Fig. 2d) [33]. Figure $2 \mathrm{e}$ shows a broadened characteristic peak at $530.8 \mathrm{eV}$, assigning to the $\mathrm{O}$ section of the heterojunction in the $\mathrm{O}$ $1 \mathrm{~s}$ spectrum [34]. In Figure S2, the Raman spectrum of 20-MWCNT/Bi-BiOl-0.5 and Bi-BiOl-0.5 also indicates that MWCNT is successfully introduced into 20-MWCNT/Bi$\mathrm{BiOl}-0.5$. All the findings are indicating that the composite is coexisted with MWCNT, Bi and BiOI.

The morphology of Bi-BiOl-0.5 and 20-MWCNT/BiBiOI- 0.5 composites were characterized by SEM. Figure $3 a$ offers a clearly expressession that $\mathrm{Bi}-\mathrm{BiOl}$ is composed of uniform interpretation made up of a microsphere structure. These microstructures featured by 20-MWCNT/BiBiOl-0.5 are shown in Fig. 3b. It is obvious that MWCNT belong to the $\mathrm{Bi}-\mathrm{BiOI}-0.5$ microspheres. Figure $3 \mathrm{c}$ displays the TEM image of 20-MWCNT/Bi-BiOI-0.5. The HRTEM examination displays three lattices spacing of $0.267 \mathrm{~nm}$, $0.285 \mathrm{~nm}$ and $0.301 \mathrm{~nm}$, which conforms to $\mathrm{Bi}(110)$, MWCNT (002) and BiOI (102) in Fig. 3d. All of the findings reveal that that the MWCNT/Bi-BiOl was given successful preparation.

The absorption attributes harbored by $\mathrm{BiOI}, \mathrm{Bi}-\mathrm{BiOI}-0.5$ and 20-MWCNT/Bi-BiOl-0.5 was put under analysis by UV-Vis DRS of Fig. 4a. BiOl interpreted an absorption edge nearly $600 \mathrm{~nm}$ in the seen region. Additionally, it could be observed clearly that Bi-BiOI and 20-MWCNT/Bi-BiOI-0.5 are featured by higher-level absorption compared to $\mathrm{BiOI}$ within the range of visible light of $400-600 \mathrm{~nm}$. Besides, 20-MWCNT/Bi-BiOl-0.5 is featured by dramatically higher than visible light absorption capacity than BiOI. Based on the fundamental electronegativity conception [35], the band gap energy is calculated in virtue of Eq. (1).

$\alpha h \nu=A(\nu h-E g) n / 2$

In the situation that $\mathrm{h}$ is Planck's constant, $v$ refers to incident light frequency, A means constant, and $\mathrm{n}$ stands for the sort of optical transition, $\mathrm{BiOl}$ refers to a direct semiconducting part, so $\mathrm{n}$ reaches 1 [36]. As Fig. $4 \mathrm{~b}$ shows, Eg of BiOl is about $1.71 \mathrm{eV}$. Subsequently, Fig. 4c further studies the ability of synthetic materials, aiming to isolate the electrons and holes by PL spectra. 20-MWCNT/Bi-BiOI-0.5 is characterized by lower intensity than $\mathrm{BiOl}$, which suggests 20-MWCNT/Bi-BiOI-0.5 having low electron recombination rate. Figure $4 \mathrm{~d}$ further reveals the charge carrier transfer properties in virtue of the time-resolved $\mathrm{PL}$ spectrum [37]. They reveal that 20-MWCNT/Bi-BiOI-0.5 has wonderful charge carrier transfer attributes, $0.91,1.01$ and $1.92 \mathrm{~ns}$ represented the lifetime of $\mathrm{BiOI}, \mathrm{Bi}-\mathrm{BiOl}-0.5$ and 20-MWCNT/Bi-BiOI-0.5. In accordance with the discussion above $20-\mathrm{MWCNT} / \mathrm{Bi}-\mathrm{BiOI}-0.5$ possesses best PEC performance.

\subsection{Photoelectrochemical sensor}

Figure 5 explains the photocurrent performance of $\mathrm{BiOl}, \mathrm{Bi}-$ $\mathrm{BiOl}-0.5$ as well as $20-\mathrm{MWCNT} / \mathrm{Bi}-\mathrm{BiOl}-0.5$ with the addition of $200 \mathrm{ng} \mathrm{mL}^{-1} 4-\mathrm{CP}$ under visible light excitation in $0.1 \mathrm{M}$ PBS at $0 \mathrm{~V}$ versus SCE. The photocurrent of all materials is increased correspondingly after adding 4-CP. Meanwhile, 20-MWCNT/Bi-BiOI-0.5 photocurrent value is much higher than other materials, attributing to that the SPR of metal $\mathrm{Bi}$ and the excellent electrical conductivity of MWCNT significantly enhanced the PEC efficiency for 4-CP $[27,30]$.

To evaluate the PEC performance given by the 20-MWCNT/Bi-BiOI-0.5 for 4-CP, photocurrent tests were carried out on different concentrations of 4-CP. After adding different concentrations of 4-CP, it can be seen in Fig. $6 \mathrm{a}$ that the photocurrent rises sharply, and Fig. $6 \mathrm{~b}$ plots the linear relationship of the photocurrent with different concentrations. Figure $6 \mathrm{~b}$ displays two linear connections of the sensor on the basis of 20-MWCNT/Bi-BiOl-0.5 for 4-CP. From 1 to $200 \mathrm{ng} \mathrm{mL}^{-1}$, the linear regressing equation means $\Delta \mathrm{I}=0.6591+0.0131 \mathrm{c}\left(\mathrm{R}^{2}=0.9970\right)$, and within the scope of $400-12,800 \mathrm{ng} \mathrm{mL}^{-1}$, the linear relationship is $\Delta \mathrm{I}=5.6146+9.914 \times 10^{-4} \mathrm{C}\left(\mathrm{R}^{2}=0.9933\right) .0 .65 \mathrm{ng} \mathrm{mL}^{-1}$ $(\mathrm{S} / \mathrm{N}=3)$ stands for the detection limit of the sensor. Moreover, Table 1 interprets a comparison of 20-MWCNT/ $\mathrm{Bi}-\mathrm{BiOl}-0.5$ with other $4-\mathrm{CP}$ sensors in the literature reports. For electrochemical, the PEC sensor of 20-MWCNT/Bi$\mathrm{BiOl}-0.5$ have the low detection limit, for other PEC sensor, we have wide linear range. Evidently, our sensor is better than other 4-CP sensors in terms of some aspect. 

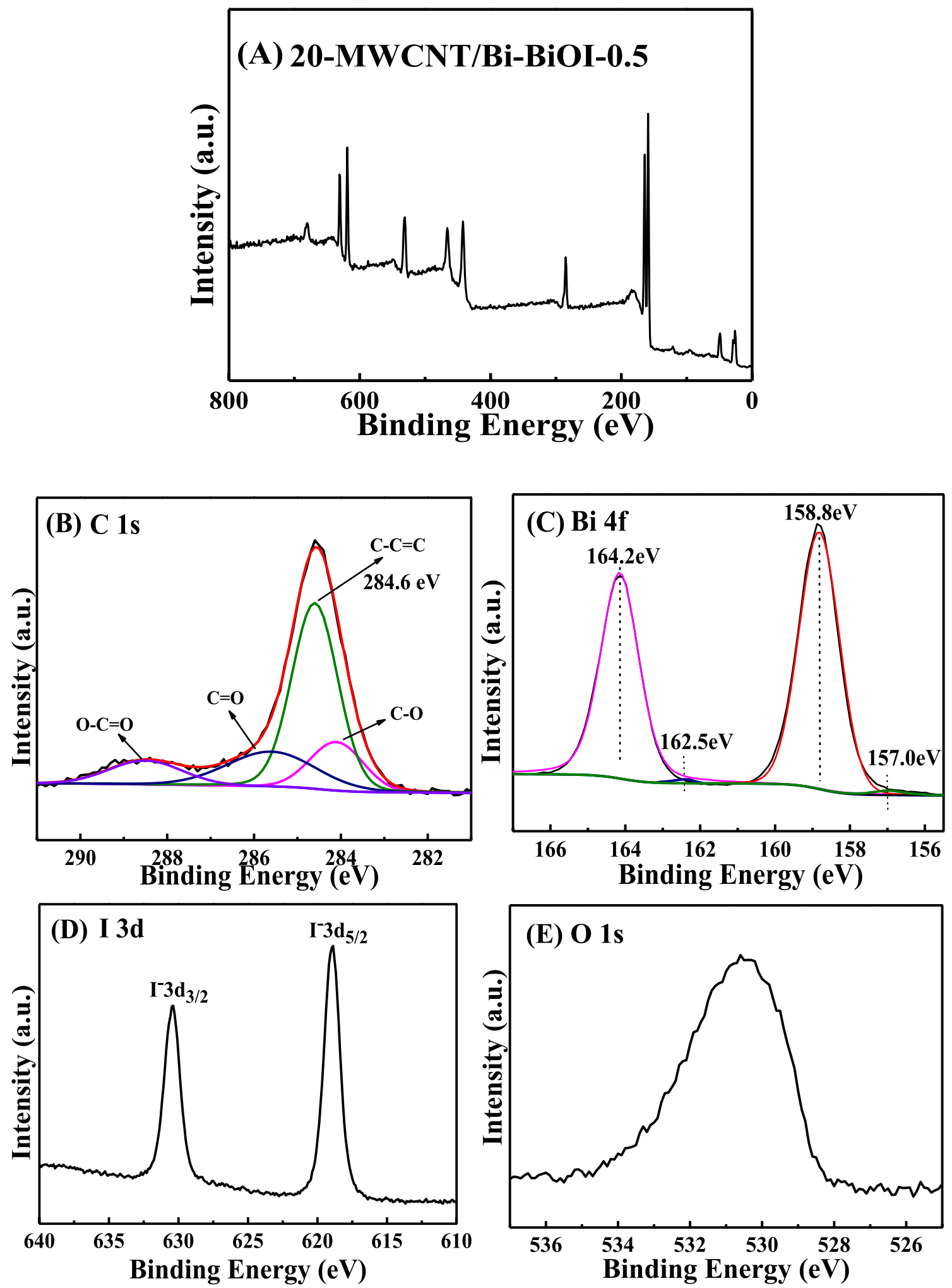

Fig. 2 XPS spectrum of a 20-MWCNT/Bi-BiOl-0.5 composites: b C 1s, c Bi 4f, d I 3d, e O 1 s 

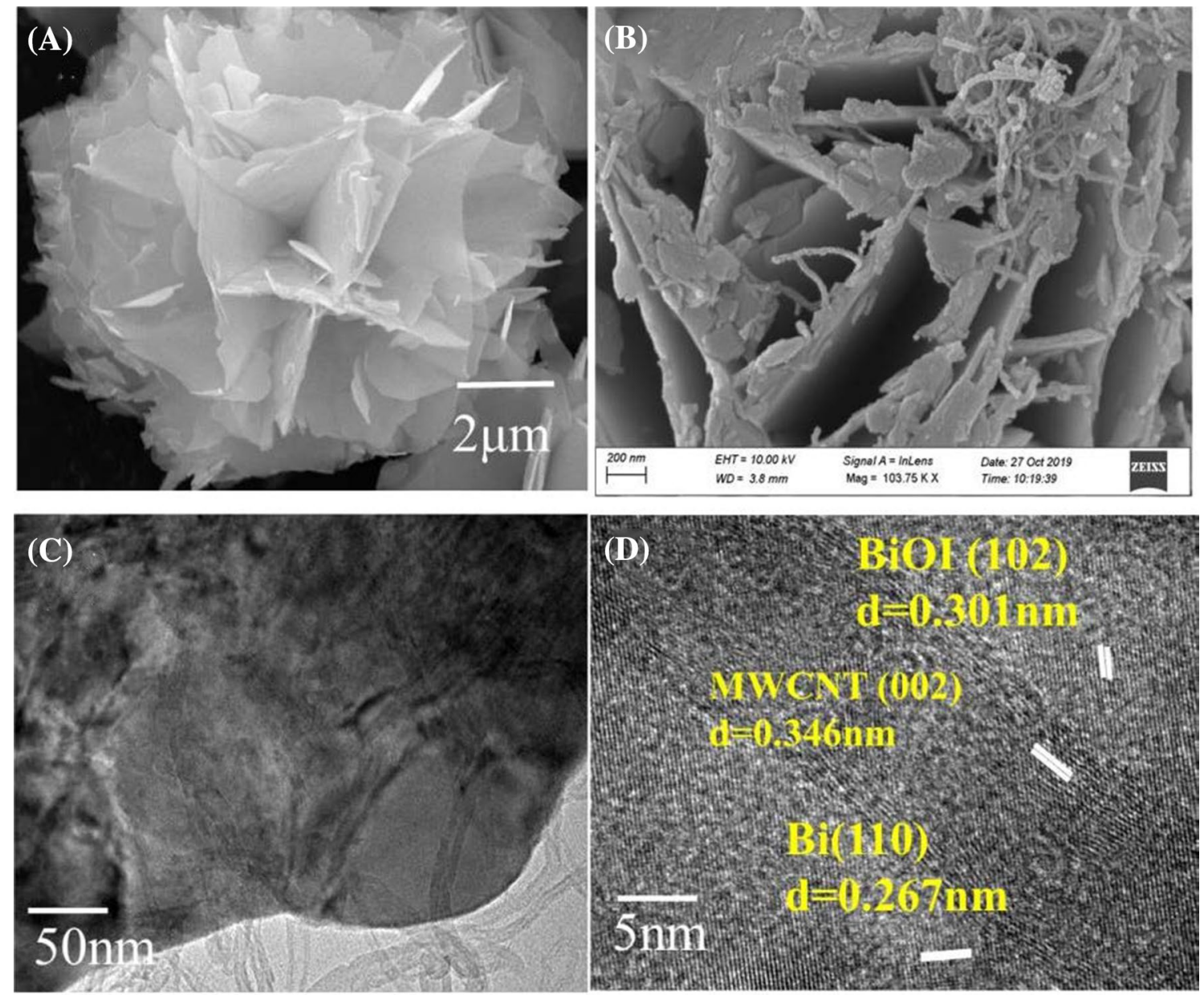

Fig. 3 SEM images of Bi-BiOI (a), 20-MWCNT/Bi-BiOI-0.5 (b); c TEM and d HR-TEM images of 20-MWCNT/Bi-BiOI-0.5

The stability harbored by $20-\mathrm{MWCNT} / \mathrm{Bi}-\mathrm{BiOI}-0.5$ was put under examination in virtue of monitoring the photocurrent of repeatedly made photoexcitation above $900 \mathrm{~s}$ (Fig. 6C). And the reaction photocurrent of 20-MWCNT/Bi-BiOl-0.5 remained $95.7 \%$ of its initial value towards $200 \mathrm{ng} \mathrm{mL}^{-1} 4$-CP within 21 days (Fig. $6 \mathrm{~d}$ ). The reproducibility featured by $20-\mathrm{MWCNT} / \mathrm{Bi}-\mathrm{BiOI}-0.5$ was tested through detecting $200 \mathrm{ng} \mathrm{mL}^{-1} 4-\mathrm{CP}$ by five parallel electrodes (Fig. 6e), as well as the photocurrents shows no evident changes, which indicates its satisfying reproducibility. The photocurrent reactions of 20-MWCNT/Bi-BiOl-0.5 towards $200 \mathrm{ng} \mathrm{mL}^{-1} 4-\mathrm{CP}$ and interfering substance were expressed in Fig. $6 \mathrm{f}$, the photocurrent responses of interferences are of negligibility. The holes of 20-MWCNT/Bi-BiOI-0.5 can reduce 4-CP, which can electrode can accelerate the charge separation and suppress the electron-hole recombination, leading to the increment of photocurrent intensity. According to this mechanism, the 20-MWCNT/Bi-BiOI-0.5 $\mathrm{PEC}$ sensor has an acceptable anti-interference capacity for $4-C P$ detection $[39,40]$.

\section{SN Applied Sciences}

To validate the practical reliability harbored by the ready-made 4-CP sensor, the combination of tap water with lake water which contain 4-CP were employed as an authentic I sample. The findings are demonstrated in Table 2, the RSD are lower than $4.7 \%$, the recoveries discovered as $99.82-100.60 \%$. Those findings demonstrate that the sensor, which is based upon 20-MWCNT/Bi-BiOI-0.5, is of reliability for the detection of 4-CP in authentic samples.

\subsection{Mechanism of PEC sensing 4-CP}

In terms of investigation on mechanism of PEC sensor of 4-CP according to MWCNT-M, $\mathrm{E}_{\mathrm{VB}}$ and $\mathrm{E}_{\mathrm{CB}}$ of and BiOI are explored with the assistance of the following equation [42]:

$E V B=X-E e+0.5 E g$

$E C B=E V B-E g$ 

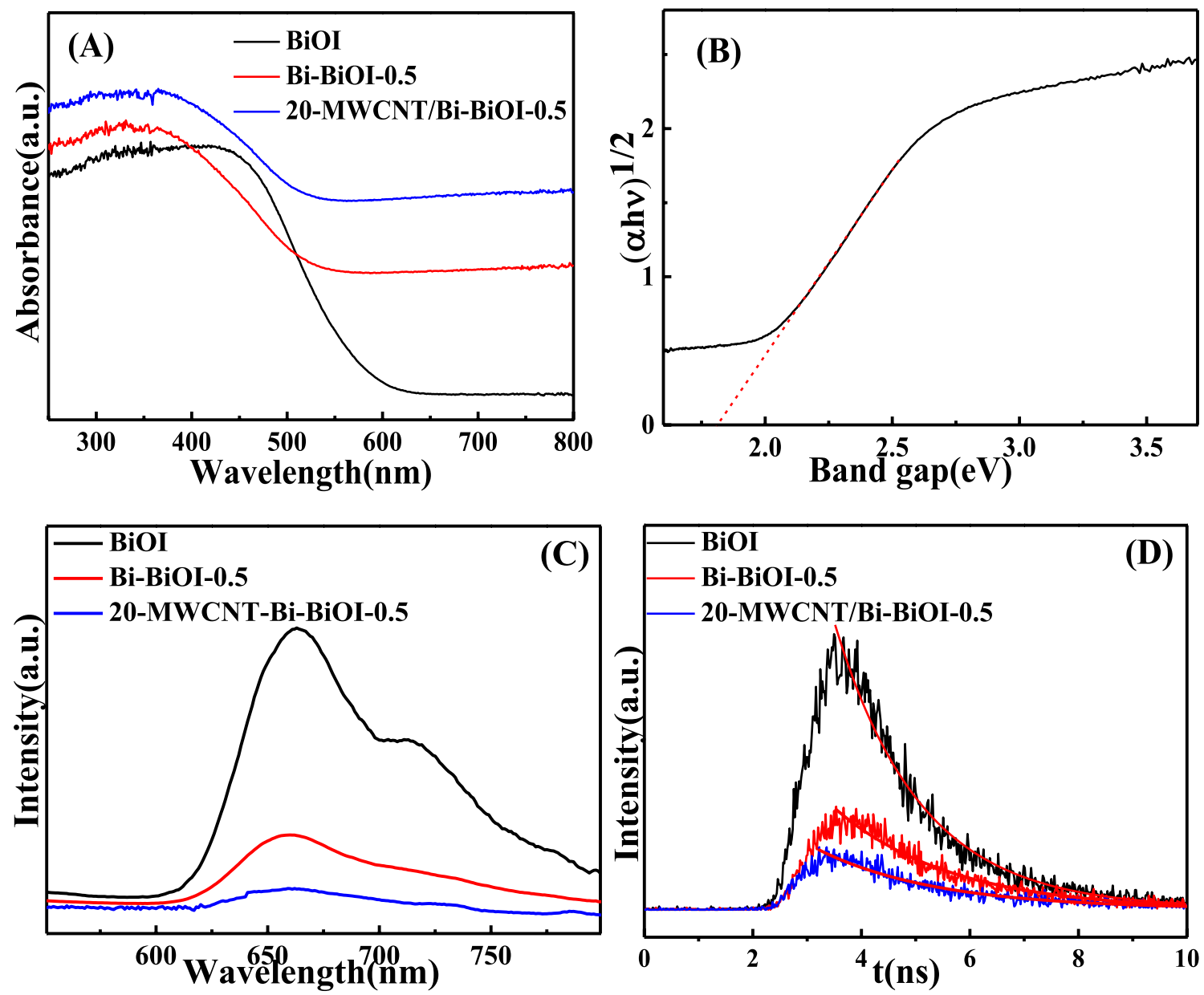

Fig. 4 UV-Vis diffuse reflectance spectrum (a); plots of (Ahv) ${ }^{1 / 2}$ versus photon energy (hv) for BiOl (b); PL spectrum (c) and the time-resolved PL spectrum (d) of materials

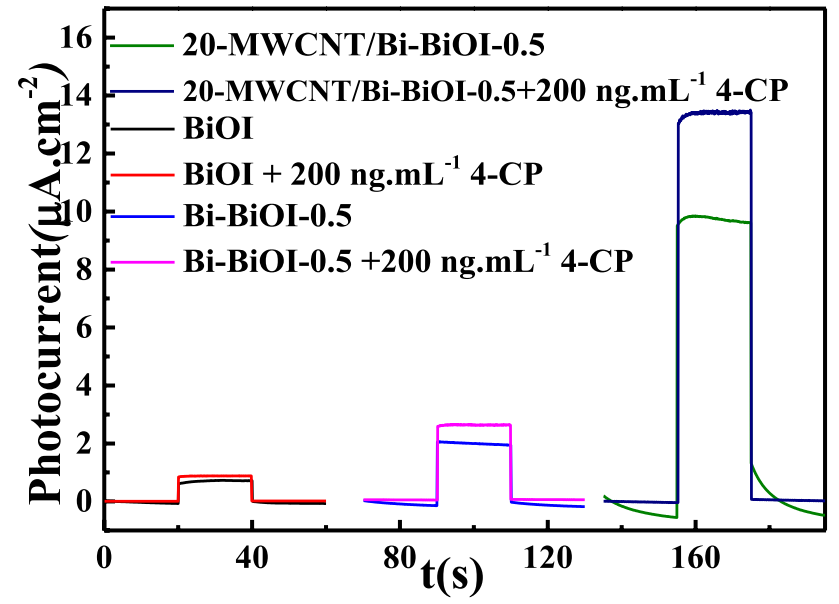

Fig. 5 Photocurrent responses of the materials in the absence and presence $200 \mathrm{ng} \mathrm{mL}^{-1} 4-\mathrm{CP}$ determination in $0.1 \mathrm{M}$ PBS at $0 \mathrm{~V}$ versus SCE with visible light excitation
Ee reaches nearly $4.5 \mathrm{eV}$, indicating the energy powered by free electrons at the hydrogen level. $X$ indicates semiconductor's electronegativity, and the $X$ value of BiOl reached $6.49 \mathrm{eV}$. The $\mathrm{CB}$ edge potentials of $\mathrm{BiOl}$ reached $0.62 \mathrm{eV}$. Besides, we found the VB edge potentials attached to $\mathrm{BiOl}$ as $+2.30 \mathrm{eV}$. Accordingly, PEC system of MWCNT/ $\mathrm{Bi}-\mathrm{BiOl}$ is schematically portrayed as the Fig. 7 show. While the compound is on illumination, electrons are able to be triggered from the $\mathrm{E}_{\mathrm{VB}}$ of $\mathrm{BiOl}$ to generating photoinduced electron-hole pairs. When the Bi nanoparticles with MWCNT got connected to BiOl microspheres, the metal-semiconductor junction, along with heterojunction was taken into formation in the interfaces of $\mathrm{Bi}-\mathrm{BiOl}$ and MWCNT/BiOl separately. What was worth mentioning is that $\mathrm{Bi}$ nanoparticles on $\mathrm{BiOl}$ microspheres could serve as light collectors in the compound. After irradiation of the visible light, electron-hole pairs were set up in the $\mathrm{Bi}$ nanoparticles as well as a result of the active SPR influence. Next, the hot electron was to transport to the $E_{C B}$ of $\mathrm{BiOI}$. In terms of high charge carrier mobility, MWCNT undertakes 

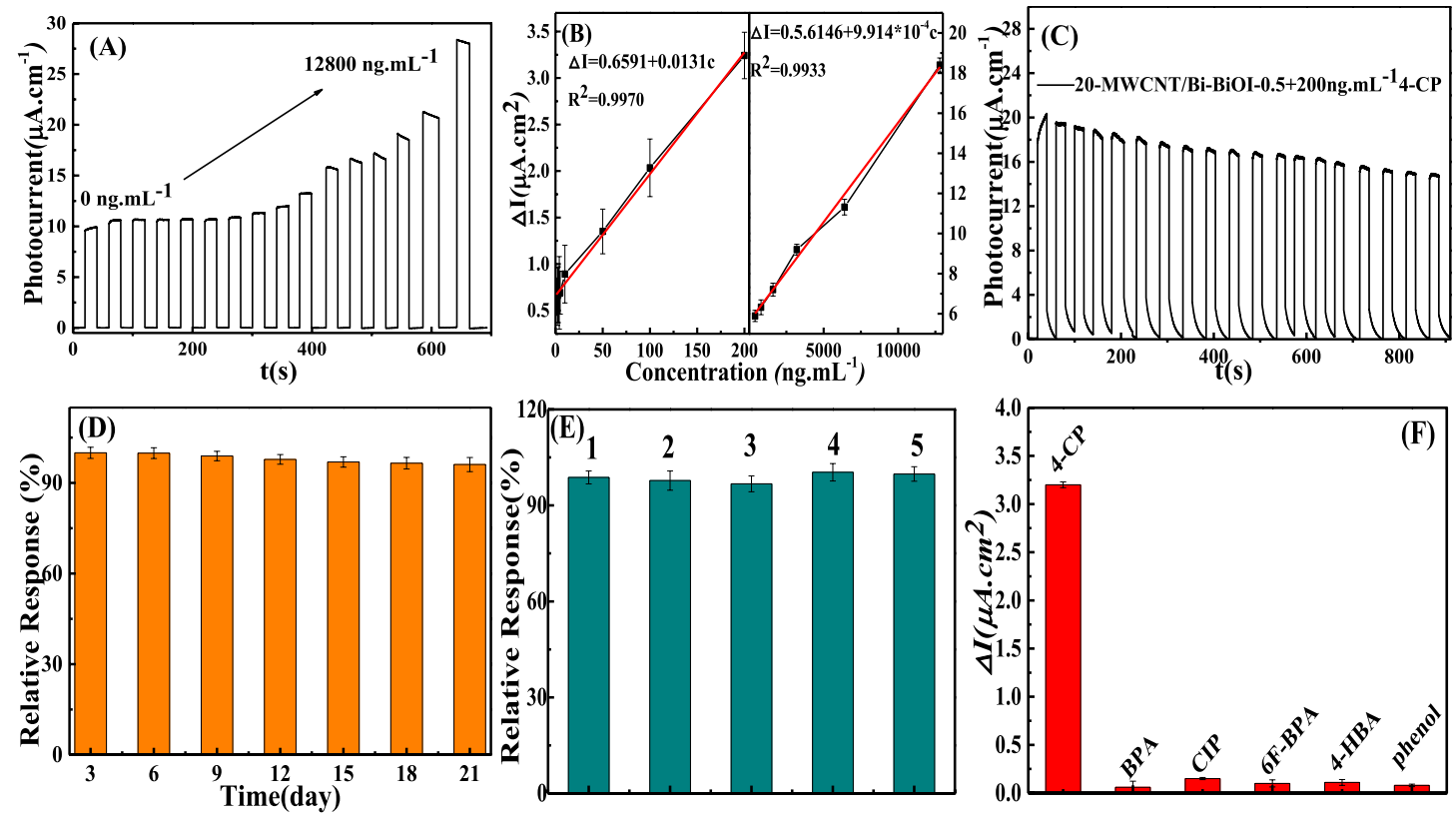

Fig. 6 Photocurrent responses of 20-MWCNT/Bi-BiOl-0.5 toward 4-CP under increasing concentrations (a), the corresponding calibration plots of the 4-CP concentration (b), stable photocurrent response of 20-MWCNT/Bi-BiOl-0.5 in the presence of $200 \mathrm{ng} \mathrm{mL}^{-1}$ $4-\mathrm{CP}(\mathbf{c})$, stable photocurrent response of $20-\mathrm{MWCNT} / \mathrm{Bi}-\mathrm{BiOl}-0.5$ in the presence of $200 \mathrm{ng} \mathrm{mL}^{-1} 4-\mathrm{CP}(\mathbf{d})$, the current responses of five-paralleled (E), Influence of interferences on $200 \mathrm{ng} \mathrm{mL}^{-1} 4-\mathrm{CP}$ determination in $0.1 \mathrm{M}$ PBS at $0 \mathrm{~V}$ versus SCE with visible light excitation (f)
Table 1 Comparison of different sensors for 4-CP assay

\begin{tabular}{lcll}
\hline Detection method & Linear range $(\mathrm{ng} / \mathrm{mL})$ & $\begin{array}{l}\text { Detection limit } \\
(\mathrm{ng} / \mathrm{mL})\end{array}$ & References \\
\hline Electrochemical detection & $103-12,900$ & 38.6 & {$[38]$} \\
Electrochemical detection & $1280-38,500$ & 474 & {$[6]$} \\
Amperometric detection & $321-5140$ & 50.1 & {$[39]$} \\
& $8040-15,100$ & & {$[40]$} \\
Voltammetric & $38.57-51,424$ & 14.14 & {$[41]$} \\
Photoelectrochemical detection & $129-1930$ & 10.3 & {$[42]$} \\
Photoelectrochemical detection & $20-33,800$ & 0.00678 & This work \\
Photoelectrochemical detection & $1-200$ & 0.65 & \\
& $400-12,800$ & & \\
\hline
\end{tabular}

Table 2 PEC detection characterized by 4-CP in lake water samples

\begin{tabular}{lrrrlr}
\hline Sample & Supplied & Discovered & Recovery (\%) & RSD (\%) & HPLC \\
\hline 1 & 1.00 & 1.06 & 100.60 & 2.7 & 1.00 \\
2 & 50.00 & 49.91 & 99.82 & 2.5 & 50.03 \\
3 & 200.00 & 200.06 & 100.03 & 4.1 & 199.97 \\
4 & 1000.00 & 999.82 & 99.98 & 3.6 & 1000.11 \\
5 & 8000.00 & 8003.12 & 100.04 & 4.7 & 8000.08 \\
\hline
\end{tabular}

to be an electron acceptor as well as a transporter to effectively stop the reintegration occurring to the photogenerated electron-hole pairs. Thereby, the lifespan of photo-generated charge carriers could be dramatically promoted in the duration of the process, thus bringing about a vastly upgraded photocatalytic effect amidst the irradiation of visible light, as the comparison with pure $\mathrm{BiOI}$. When 4-CP introduction occurs, the holes within the BiOl valence band (VB) could be defeated by 4-CP, which can electrode can accelerate the charge separation and suppress the electron-hole recombination, leading to the increment of photocurrent intensity. This kind of synergy effect in the MWCNT/Bi-BiOl electrode is able to speed up the charge separation by suppressing the electron-hole reintegration that contributes to the growth of photocurrent intensity. 
Fig. 7 PEC mechanism of MWCNT/Bi-BiOl for the detection of $4-\mathrm{CP}$

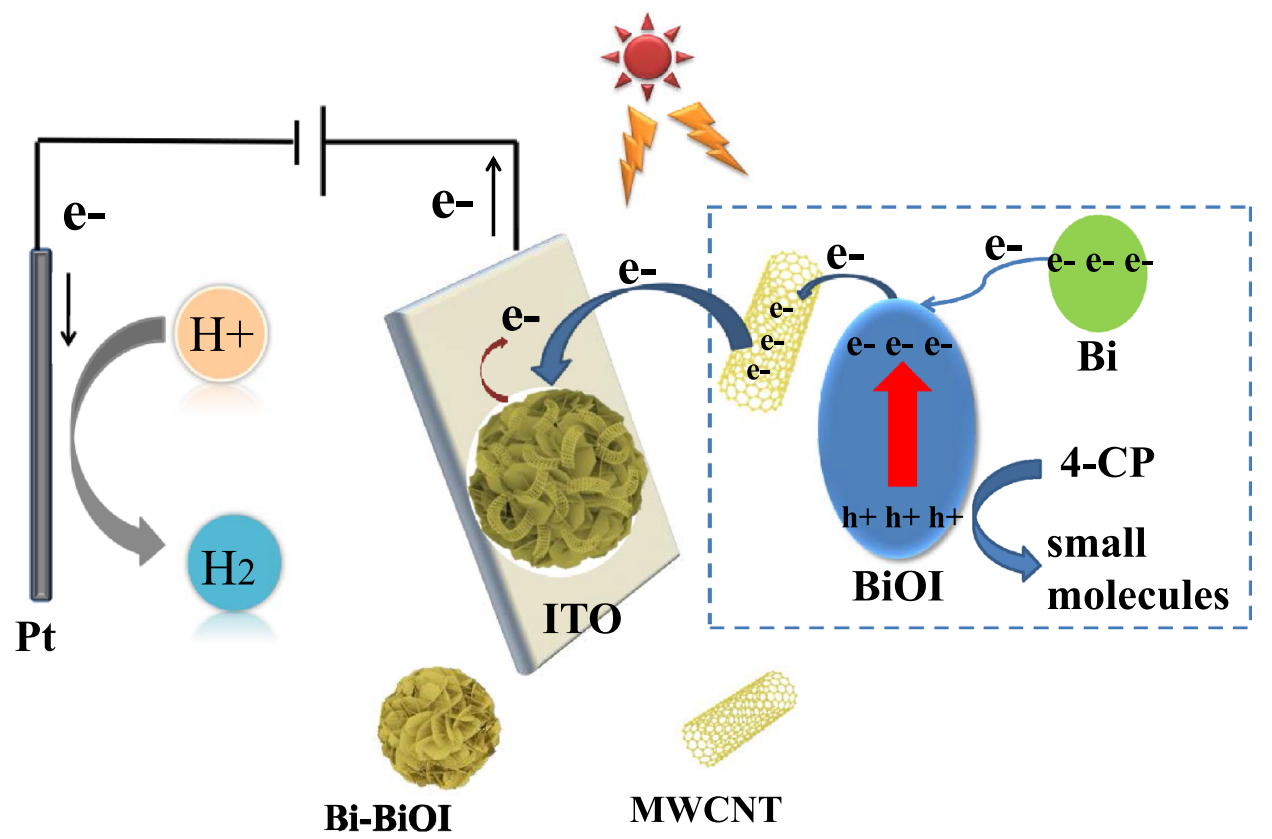

\section{Conclusions}

In summary, the PEC method based on MWCNT/Bi-BiOI was developed to detect exhibit 4-CP, introducing metal $\mathrm{Bi}$ and MWCNT is a well-functioning approach to improving the assaying properties. The findings expressed better photoelectrocatalytic active due to metal $\mathrm{Bi}$ and MWCNT strengthened the degree of light absorption as well as reducing the reintegration ratio of the electron holes. The study demonstrated the potential application of MWCNT/Bi-BiOl to 4-CP photoelectrochemical detection.

Acknowledgements This work is supported by the Applied Basic Research Youth Science and Technology Foundation of Shanxi Province of China (No. 201901D211433), the Natural Science Foundation of Shanxi Province of China (No. 201801D121073), the Scientific and Technological Innovation Programs of Higher Education Institutions in Shanxi Province (No. 2019L0737), the Scientific and Technological Innovation Programs of Higher Education Institutions in Shanxi Province (No. 2019L0749), 2019 Open Research Fund of Shanxi Province Research Center for Innovative Application of New Mesoporous Materials (No. MMIA2019106), The Key projects of science and technology research in Hebei higher education institutions (ZD2018311), Xingtai young science and Technology Talents Project (2019ZZ023), Xingtai science and technology program (2019ZC007, 2018ZC031, 2018ZC227).

\section{Compliance with ethical standards}

Conflict of interest The authors have no conflicts of interest to declare.

\section{References}

1. Yang Z, Zhang X, Pu SJ, Ni RX, Lin Y, Liu Y (2019) Novel Fentonlike system (Mg/Fe-O2) for degradation of 4-chlorophenol. Environ Pollut 250:906-913

2. Guzmán IC, Rodríguez JL, Poznyak T, Chairez I, Hernández I, Hernández RT (2020) Catalytic ozonation of 4-chlorophenol and 4-phenolsulfonic acid by $\mathrm{CeO}_{2}$ films. Catal Commun 133:105827

3. Wei Y, Zhang YW, Geng W, Su HR, Long MC (2019) Efficient bifunctional piezocatalysis of Au/BiVO4 for simultaneous removal of 4-chlorophenol and $\mathrm{Cr}(\mathrm{VI})$ in water. Appl Catal $\mathrm{B}$ Environ 259:118084

4. Galán-Cano F, Lucena R, Cárdenas S, Valcárcel M (2012) lonic liquid based in situsolvent formation microextraction coupled to thermal desorption forchlorophenols determination in waters by gas chromatography/massspectrometry. J Chromatogr A 1229:48-54

5. Alizadeh R (2016) Chlorophenol's ultra-trace analysis in environmental samples bychitosan-zinc oxide nanorod composite as a novel coating for solid phasemicro-extraction combined with high performance liquid chromatography. Talanta 146:831-838

6. Qiu CC, Chen T, Wang X, Li Y, Ma HY (2013) Application of horseradish peroxidase modified nanostructured $\mathrm{Au}$ thin films for the amperometric detection of 4-chlorophenol. Colloids Surf B 103:129-135

7. Sheng PT, Yao L, Yang P, Yang DF, Lu CK, Cao KS, Li WL (2020) The origin of enhanced photoelectrochemical activity in metal-iondoped $\mathrm{ZnO} / \mathrm{CdS}$ quantum dots. J Alloys Compd 822:153700

8. Xu SM, Jiang JG, Ren WY, Wang H, Zhang R, Xie YG, Chen YB (2020) Construction of $\mathrm{ZnO} / \mathrm{CdS}$ three-dimensional hierarchical photoelectrode for improved photoelectrochemical performance. Renew Energy 153:241-248

9. Scherrer B, Li T, Tsyganok A, Döbeli M, Gupta B, Malviya KD, Kasian O, Maman N, Gault B, Grave DA, Mehlman A, VisolyFisher I, Raabe D, Rothschild A (2020) Defect segregation and 
its effect on the photoelectrochemical properties of Ti-doped hematite photoanodes for solar water splitting. Chem Mater 32:1031-1040

10. Wang B, Cao JT, Liu YM (2020) Recent progress of heterostructure-based photoelectrodes in photoelectrochemical biosensing: a mini review. Analyst 145:1121-1128

11. Yu ZY, Detlef B, Ralf D, Song L, Lu LQ (2012) Photocatalytic degradation of azo dyes by $\mathrm{BiOX}(\mathrm{X}=\mathrm{Cl}, \mathrm{Br})$. J Mol Catal $\mathrm{A}$ Chem 365:1-7

12. Lv JX, Hu QS, Cao CJ, Zhao YP (2018) Modulation of valence band maximum edge and photocatalytic activity of BiOX by incorporation of halides. Chemosphere 191:427-437

13. Jiang $Z Y$, Huang $B B$, Lou ZZ, Wang ZY, Meng XD, Liu YY, Qin $X Y$, Zhang $X Y$, Dai $Y$ (2014) Immobilization of $\mathrm{BiOX}(X=\mathrm{Cl}, \mathrm{Br})$ on activated carbon fibers as recycled photocatalysts. Dalton Trans 43:8170-8173

14. Yang $Y$, Zhang $C$, Lai $C$, Zeng $G M$, Huang $D L$, Cheng $M$, Wang JJ, Chen F, Zhou CY, Xiong WP (2018) BiOX (X =Cl, Br, I) photocatalytic nanomaterials: applications for fuels and environmental management. Adv Colloid Interfaces 254:76-93

15. Ye LQ, Su YR, Jin XL, Xie HQ, Zhang C (2014) Recent advances in $\mathrm{BiOX}(\mathrm{X}=\mathrm{Cl}, \mathrm{Br}$ and I) photocatalysts: synthesis, modification, facet effects and mechanisms. Environ Sci Nano 1:90-112

16. Hu XC, Wang GH, Wang J, Hu ZF, Su YR (2020) Step-scheme NiO/ $\mathrm{BiOI}$ heterojunction photocatalyst for rhodamine photodegradation. Appl Surf Sci 511:145499

17. Yuan $D$, Huang LY, Li YP, Wang H, Xu XQ, Wang CB, Yang L (2020) A novel Agl/BiOl/pg-C3N4 composite with enhanced photocatalytic activity for removing methylene orange tetracycline and E. coli. Dyes Pigments 177:108253

18. Tian N, Huang HW, Wang SB, Zhang TR, Du X, Zhang YH (2020) Facet-charge-induced coupling dependent interfacial photocharge separation: a case of $\mathrm{BiOl} / g-C 3 N 4$ p-n junction. Appl Catal B Environ 267:118697

19. Liu HH, Yang C, Huang J, Chen JF, Zhong JB, Li JZ (2020) lonic liquid-assisted hydrothermal preparation of $\mathrm{BiOl} / \mathrm{BiOCl}$ heterojunctions with enhanced separation efficiency of photo-generated charge pairs and photocatalytic performance. Inorg Chem Commun 113:107806

20. Li JH, Yang F, Zhou Q, Wu LJ, Li WY, Ren RP, Yk Lv (2020) Correction: visible-light photocatalytic performance, recovery and degradation mechanism of ternary magnetic $\mathrm{Fe}_{3} \mathrm{O}_{4} / \mathrm{BiOBr} / \mathrm{BiOl}$ composite. RSC Adv 10:1828

21. Zhong S, Wang BQ, Zhou H, Li CY, Peng XJ, Zhang SY (2019) Fabrication and characterization of $\mathrm{Ag} / \mathrm{BiOl} / \mathrm{GO}$ composites with enhanced photocatalytic activity. J Alloys Compd 806:401-409

22. Wang YQ, Jiang S, Liu F, Zhao CC, Zhao DF, Li XF (2019) Study on preparation and toluene removal of $\mathrm{BiOl} / \mathrm{Bi}_{2} \mathrm{WO}_{6} / \mathrm{ACF}$ photocatalyst. Appl Surf Sci 488:161-169

23. Hu CC, Huang HX, Lin YF, Tung KL, Chen TH, Lo L (2019) Heterostructural design of I-deficient $\mathrm{BiOl}$ for photocatalytic decoloration and catalytic $\mathrm{CO}_{2}$ conversion. Catal Sci Technol 9:3800-3811

24. Lv JX, Liu XM, Li PC, Jin W, Xu J, Zhao YP (2019) Agl loading $\mathrm{BiOl}$ composites with enhanced photodegradation efficiency for bisphenol A under simulated solar light. Sci Total Environ 669:194-204

25. Ma Y, Jia YL, Wang L, Yang M, Bi YP, Qi YX (2016) Efficient charge separation between $\mathrm{Bi}$ and $\mathrm{Bi}_{2} \mathrm{MoO}_{6}$ for photoelectrochemical properties. Chem Eur J 22:5844-5848

26. Li MY, Zhang GX, Feng CQ, Wu HM, Mei H (2020) Highly sensitive detection of chromium (VI) by photoelectrochemical sensor under visible light based on Bi SPR-promoted BiPO4/BiOI heterojunction. Sensor Actuator B Chem 305:127449

27. Jing QF, Feng XY, Pan JL, Chen LM, Liu YN (2018) Facile synthesis of $\mathrm{Bi} / \mathrm{BiVO}_{4}$ composite ellipsoids with high photocatalytic activity. Dalton Trans 47:2602-2609
28. Zhao XF, Zhang J, Wang XQ, Zhang J, Liu BX, Yi XB (2019) Polyimide aerogels crosslinked with MWCNT for enhanced visible-light photocatalytic activity. Appl Surf Sci 478:266-274

29. Liu D, Xie JH, Xia Y (2019) Improved photocatalytic activity of MWCNT/BiOBr composite synthesized via interfacial covalent bonding linkage. Chem Phys Lett 729:42-48

30. Zhang X, Wang XB, Wang LM, Wang WK, Long LL, Li WW, Yu HQ (2014) Synthesis of a highly efficient BiOCl single-crystal nanodisk photocatalyst with exposing 001 Facets. ACS Appl Mater Interfaces 6:7766-7772

31. Xia JX, Di J, Li HT, Xu H, Li HM, Guo SJ (2019) lonic liquid-induced strategy for carbon quantum dots/BiOX $(\mathrm{X}=\mathrm{Br}, \mathrm{Cl})$ hybrid nanosheets with superior visible light-driven photocatalysis. Appl Catal B 18:260-269

32. Jiang J, Zhang X, Sun PB, Zhang LZ (2011) ZnO/BiOl heterostructures: photoinduced charge-transfer property and enhanced visible-light photocatalytic activity. J Phys Chem C 115:20555-20564

33. Yan PC, Jiang DS, Li HN, Cheng M, Xu L, Qian JC, Bao J, Xia JX, Li HM (2018) Exploitation of a photoelectrochemical sensing platform for catechol quantitative determination using $\mathrm{BiPO}_{4}$ nanocrystals/BiOl heterojunction. Anal Chim Acta 1042:11-19

34. Kuo WS, Ho PH (2006) Solar photocatalytic decolorization of dyes in solution with $\mathrm{TiO}_{2}$ film. Dyes Pigments 71:212-217

35. Li B, Chen XW, Zhang TY, Jiang S, Zhang GH, Wu WB, Ma XY (2018) Photocatalytic selective hydroxylation of phenol to dihydroxybenzene by $\mathrm{BiOl} / \mathrm{TiO} 2 \mathrm{p}-n$ heterojunction photocatalysts for enhanced photocatalytic activity. Appl Surf Sci 439:1047-1056

36. Cao J, Xu BY, Lin HL, Chen SF (2013) Highly improved visible light photocatalytic activity of $\mathrm{BiPO}_{4}$ through fabricating a novel $\mathrm{p}-\mathrm{n}$ heterojunction $\mathrm{BiOl} / \mathrm{BiPO}_{4}$ nanocomposite. Chem Eng J 228:482-488

37. Wang B, Okoth OK, Yan K, Zhang JD (2016) A highly selective electrochemical sensor for 4-chlorophenol determination based on molecularly imprinted polymer and PDDA-functionalized graphene. Sensor Actuator B Chem 236:294-303

38. Zhu XL, Zhang KX, Wang DW, Zhang DM, Yuan X, Qu J (2018) Electrochemical sensor based on hydroxylated carbon nanotubes/platinum nanoparticles/rhodamine B composite for simultaneous determination of 2,4,6-trichlorophenol and 4-chlorophenol. J Electroanal Chem 810:199-206

39. Wang L, Sun Q, Liu Y, Lu ZS (2016) Voltammetric determination of 4-chlorophenol using multiwall carbon nanotube/gold nanoparticle nanocomposite modified glassy carbon electrodes. RSC Adv 6:34692-34698

40. Xu L, Ling SY, Li HN, Yan PC, Xia JX, Qiu JX, Wang K, Li HM, Yuan SQ (2017) Photoelectrochemical monitoring of 4-chlorophenol by plasmonic Au/graphitic carbon nitride composites. Sensor Actuator B Chem 240:308-314

41. Yan PC, Jiang DS, Li HN, Bao J, Xu L, Qian JC, Chen C, Xia JX (2019) $\mathrm{BiPO}_{4}$ nanocrystal/BiOCl nanosheet heterojunction as the basis for a photoelectrochemical 4-chlorophenol sensor. Sensor Actuator B Chem 279:466-475

42. Xu Y, Schoonen MAA (2000) The absolute energy positions of conduction and valence bands of selected semiconducting minerals. Am Miner 85:543-556

Publisher's Note Springer Nature remains neutral with regard to jurisdictional claims in published maps and institutional affiliations. 